\title{
Progressor: personalized visual access to programming problems
}

\author{
Fedor Bakalov \\ Institute for Computer \\ Science, University of Jena \\ Ernst-Abbe-Platz 2 \\ Jena 07743 , Germany \\ +493641946435
}

fedor.bakalov@uni-jena.de

\author{
I-Han Hsiao \\ School of Information \\ Sciences, University of \\ Pittsburgh \\ 135 N. Bellefield Ave. \\ Pittsburgh PA 15260, USA \\ +1 4126249437
}

ihh4@pitt.edu

\author{
Peter Brusilovsky \\ School of Information \\ Sciences, University of \\ Pittsburgh \\ 135 N. Bellefield Ave. \\ Pittsburgh PA 15260, USA \\ +1 4126249404 \\ peterb@pitt.edu
}

\author{
Birgitta König-Ries \\ Institute for Computer \\ Science, University of Jena \\ Ernst-Abbe-Platz 2 \\ Jena 07743 , Germany \\ +493641946430 \\ birgitta.koenig-ries@uni- \\ jena.de
}

\begin{abstract}
This paper presents Progressor, a visualization of open student models intended to increase the student's motivation to progress on educational content. The system visualizes not only the user's own model, but also the peers' models. It allows sorting the peers' models using a number of criteria, including the overall progress and the progress on a specific topic. Also, in this paper we present results of a classroom study confirming our hypothesis that by showing a student the peers' models and ranking them by progress it is possible to increase the student's motivation to compete and progress in e-learning systems.
\end{abstract}

Keywords - open user model; open social student model; visualization; e-learning; self-assessment;

\section{INTRODUCTION}

Recently, social learning has gained much interest in elearning systems as a technology to engage students and motivate them to learn and perform better. According to social learning theory, people can learn by what they observe from their environment [16]. The concepts of social learning can be used for modeling the desired behavior in e-learning, for instance, for motivating students to progress. Also, combined with Web 2.0 technology, social learning can provide valuable guidance through the learning content. Another approach that can support students in e-learning systems is open student modeling. Open student models provide students with a holistic and easy-to-grasp view on their knowledge [1-3]. They can help students to identify learning goals and to achieve them.

In this paper we present Progressor, a system that visualizes the student's progress on parameterized questions in the settings of a social e-learning system. The system is a successor of our earlier visualization, Parallel IntrospectiveViews $[12,13]$, which enables a student to compare his/her progress with the progress of another peer in one-to-one comparison mode. The newer version, in addition to the one-to-one comparison, allows the student to view models of multiple peers at the same time. It also allows sorting peers either by their overall progress or the progress in a certain topic and shows the student's position in the ranking. As the evaluation results show, the newer version makes a much stronger impact on the student's interaction with the learning content: Students using Progressor make more progress and have higher success rates than the ones without this visualization. The rest of this paper is organized as follows: In the next section we provide a short review of the related work on open user modeling and social learning. The system and study design are presented in the following section. Then we report the evaluation results. Finally, we summarize this work and discuss the future research plan.

\section{RELATED WORK}

There are two main streams of work on open student models. One stream focuses on visualizing the model to support students' self-reflection and planning; the other one encourages students to participate in the modeling process, such as engaging students through the negotiation or collaboration on construction of the model [2]. Representations of the student model vary from displaying high-level summaries (such as skill meters) to complex concept maps or Bayesian networks. A range of benefits have been reported on opening the student models to the learners, such as increasing the learner's awareness of the developing knowledge, difficulties and the learning process, and students' engagement, motivation, and knowledge reflection [1-3]. Dimitrova et al. [5] explore interactive open learner modeling by engaging learners to negotiate with the system during the modeling process. Chen et al. [6] investigated active open learner models in order to motivate learners to improve their academic performance. Both individual and group open learner models were studied and demonstrated the increase of reflection and helpful interactions among teammates. Bull \& Kay [7] described a framework to apply open user models in adaptive learning environments and provided many in-depth examples. In our own work on the QuizGuide system [11] we embedded open learning models into adaptive link annotation and demonstrated that this arrangement can remarkably increase student motivation to work with non-mandatory educational content.

To support social learning, it is common to show learners average values of the group model, e.g., average knowledge status of the group in a given topic. Open group modeling enables students to compare and understand their own states. 
Such group models have been used to support collaboration between learners among the same group, and to foster competition in a group of learners [8]. Vassileva and Sun [8] investigated the community visualization in online communities. They summarized that social visualization increases social interaction among students, encourages competition, and provides students the opportunity to build trust in others and in the group. Bull \& Britland [9] used OLMlets to research the problem of facilitating group collaboration and competition. The results showed that optionally releasing the models to their peers increases the discussion among students and encourages them to start working sooner. CourseVis [10] is one of the few systems providing graphical visualization for multiple groups of users to teachers and learners. It helps instructors to identify problems early on, and to prevent some of the common problems in distance learning. Therefore, it motivates us to investigate further on the social visualization techniques in the open student model systems.

\section{SYSTEM DESCRIPTION}

Progressor is intended to provide students with a holistic and easy-to-grasp view on their progress and relate it to the progress of other students in the class. The goal of the system is not only to help students in accessing the right learning content at the right time, but also to motivate them to progress and perform better. Our main hypothesis is that allowing a student to view the progress of others and compare it with their own data can motivate to progress on the learning content.

Progressor is a merger of two our earlier works, namely the QuizJET system [11] for the authoring and delivery of parameterized questions for the Java programming language and the IntrospectiveViews interface $[12,13]$ for visualization of semantic user models. The first version of Progressor was presented in [14] as Parallel IntrospectiveViews. It offers visualization of student progress on QuizJET questions of an Object-Oriented Programming course. All questions are parameterized, i.e., they include a random parameter, which QuizJET instantiates when the question is delivered to a student. As a result, the student can attempt to answer the same question multiple times with different values of the parameter, which helps to achieve the mastery level. The visualization (Fig.1) consists of two panes: the left pane displays the student's own model, whereas the right one displays someone else's model. Each pane visualizes the respective student's progress as a pie chart consisting of circular sectors representing the class lectures. The lectures are displayed in a clockwise order denoting their pre-requisite sequence, i.e., the order they are taught in class. Lectures may consist of one or several topics, which are represented as annular sectors placed within the circular sector of the corresponding lecture. The shade of each annular sector denotes whether the topic has been covered and, for the covered ones, indicates the progress the student has made with respect to the topic. The sectors painted light grey represent the topics that have not been covered yet, whereas the sectors painted a shade from the color range red to green represent the sectors that have been already covered. For the covered topics, the interface displays the student progress, i.e., the ratio of successfully completed quizzes to the total quiz count in the topic. If the ratio equals 0 , i.e., no quiz has been successfully completed, the sector is painted red. If it equals 1 , i.e., all quizzes have been completed, the sector appears green. The shades in the range between red and green denote partial completion of the quizzes. By clicking a sector, the interface will display the contents of the corresponding topic, i.e., the list of questions for the topic (Fig. 2). For each question, the interface provides a visual cue indicating the student's progress and displays the total number of attempts the student has made on the quiz and the number of successful attempts. By clicking a quiz label the interface will display the quiz in a new window.

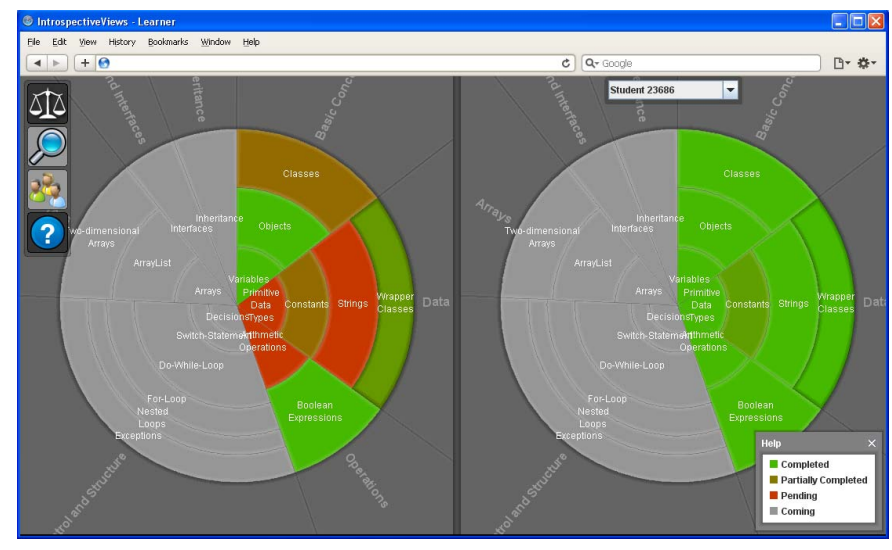

Figure 1. Parallel IntrospectiveViews (precedessor of Progressor). Left pane - visualization of the student's own progress; right pane - visualization of a peer's progress. The circular sectors represent the lectures and the annular sectors represent the topics of individual lectures. The shades of the sectors indicate whether the topic has been covered and for the covered ones, denote the progress the student has made. Color screenshots available at: http://www.minervaportals.de/research/introspective-views/.

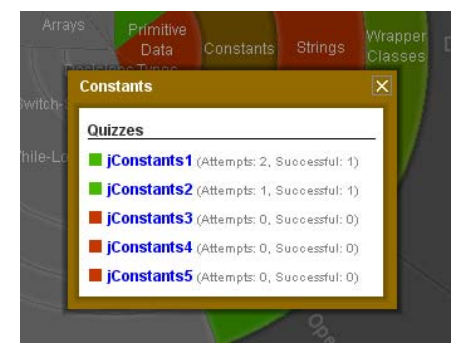

Figure 2. Parallel IntrospectiveViews. Quizzes of the selected topic.

Parallel IntrospectiveViews helped us to achieve $28 \%$ increase of the number of attempts on questions (see Sect. IV). However, we believed that by introducing a ranking feature, i.e., sorting students by progress, it would be possible to increase the competition among the students, which may also lead to an increase in the progress. To check this hypothesis we developed Progressor, a second generation of Parallel IntrospectiveViews. In the newer version (Fig. 3), the user can view the progress of multiple students at the same time and sort them in a number of ways. Next to the pie chart representing the user's own progress, the interface displays a list of thumbnails of progress charts for the other students in the class. The user can sort the peers' models by four criteria, namely, overall progress, progress in the selected topic, access to the user's progress data, and name. By choosing one of the two 
options for sorting by progress, the interface will first display the models of the top three students in the class, then the rest of open models ranked from the highest progress to the lowest, and at the end of the list it will show the models to which the user does not have access. The list of the top three students may include students whose models are open as well as the closed models. To ensure the privacy, the slices of covered topics of closed models are painted in dark grey. The sorted list rudimentarily contains the thumbnail of the user's own progress, which allows determining his/her ranking in the class with respect to either the overall progress or the progress in a selected topic. Also, the list contains a thumbnail with the average progress of the entire class. We believe that such a way for relating the student's own progress to the progress of other individual students and the class on average can be a strong motivating factor for completing more quizzes and achieving better scores.

In addition to the preview of peers' progress shown as thumbnails, the user can obtain a detailed view on the progress of an individual peer. By clicking the thumbnail of a certain student, the interface will turn into the one-to-one comparison mode (similar to the one shown in Fig. 1). In this mode, the user can obtain detailed information about the peer's progress, including the information about the progress on individual quizzes. Yet another important feature that was added in the Progressor version is the function for privacy management. The user can grant and discontinue access to his/her progress data for each peer individually. Also, it allows sending requests for access to models of other peers. The privacy settings for each peer are displayed on the peer's thumbnail as two arrows: the left arrow indicates the peer's access to the user's data and the right arrow indicates the user's access to the peer's data. The arrows may be in one of the three shades: green - access granted, red - no access, yellow - access requested. By clicking an arrow, the user can change the access status for each peer individually, e.g., by clicking the left arrow the user can grant and discontinue the peer's access to the own model. In such a way the user should be able to quickly define the desired progress sharing settings.

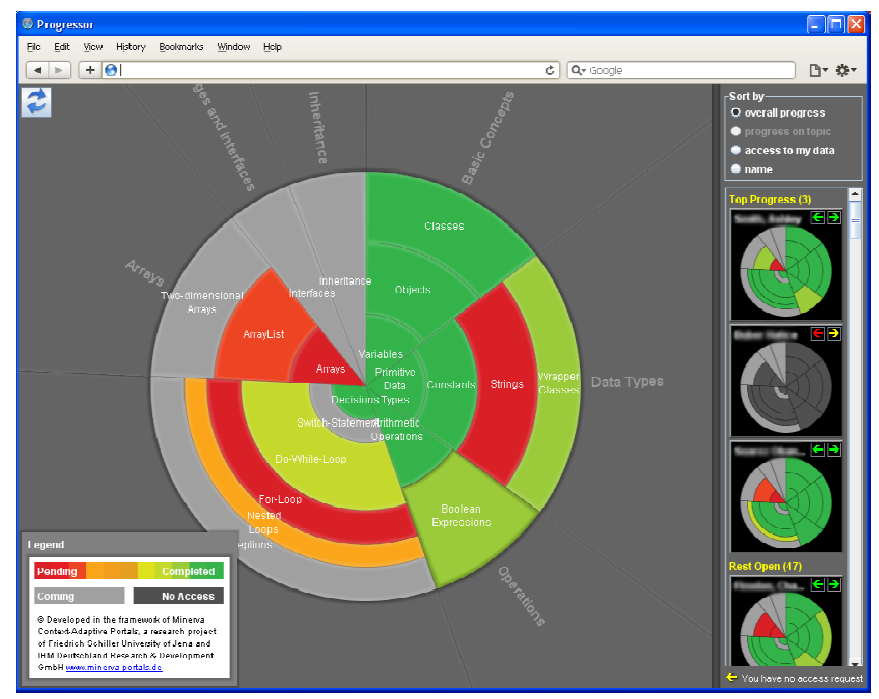

Figure 3. Progressor: Peers' progress are displayed as thumbnails and listed at the side of the user's own model.

\section{EVALUATION}

To evaluate the impact of our technology, we conduct an evaluation in a classroom study. The study was carried out in an undergraduate Object-Oriented Programming course offered by the School of Information Sciences, University of Pittsburgh in the current semester, Spring of 2011. All students received access to self-assessment quizzes through the Progressor interface. The system was introduced to the class at the third week (2011/01/20) of the course and served as the nonmandatory course tool over the complete semester period. Out of 51 students enrolled in the course, 5 of them withdrew at the moment of writing this paper; 29 out of 46 are actively using the system. All student activity with the system was recorded. For every student attempt to answer a question, the system stored a timestamp, the user's name, the question, quiz, and session ids, and the correctness of the answer.

TABLE I. SYSTEM USAGE SUMMARY

\begin{tabular}{ccccc}
\cline { 2 - 5 } & $1^{1}$ & 2 & 3 & 4 \\
\cline { 2 - 5 } & Progressor & $\begin{array}{c}\text { QuizJET w/ } \\
\text { PIV }\end{array}$ & $\begin{array}{c}\text { QuizJET } \\
\text { w/ Portal }\end{array}$ & JavGuide \\
\hline Parameters & $\mathbf{n = 2 9}$ & $\mathbf{n = 1 8}$ & $\mathbf{n = 1 6}$ & $\mathbf{n = 2 2}$ \\
\hline Attempts & $137.93 \pm 26.72$ & $113.05 \pm 15.17$ & $80.81 \pm 22.06$ & $125.50 \pm 20.04$ \\
Success Rate (\%) & $74.65 \pm 2.16$ & $71.35 \pm 3.39$ & $42.63 \pm 1.99$ & $58.31 \pm 7.92$ \\
Sessions & $5.83 \pm 0.90$ & $4.11 \pm .70$ & $3.75 \pm 0.53$ & $4.14 \pm 0.75$ \\
\hline
\end{tabular}

On average, each active user attempted 138 different questions and achieved a success rate of $74.6 \%$ on answering the questions. The data is summarized in Table 1. Following our past experience with open student modeling in JavaGuide [11], we expected the new design to view and overview the student knowledge progress would encourage the students to work more with the system. To assess it, we compared the student usage of self-assessment quizzes through the new design, Progressor (Column 1 in Tab. 1), to the older version, Parallel IntrospectiveViews (Column 2 in Tab. 1) with the data from a comparable class that accessed quizzes using a traditional a course portal with no progress visualization (Column 3 in Tab. 1) and another class accessing quizzes through an adaptive hypermedia system JavaGuide (Column 4 in Tab. 1). We found that the social visualization of student models with Parallel IntrospectiveViews caused a $39 \%$ increase of the average attempts compared to the traditional course portal. Moreover, the new design, Progressor, achieved a $70.68 \%$ increase on the average attempts. There is a significant growth on the attempts compared to the traditional course portal, $F(1,43)=4.121$, $p<.05, \eta^{2}=.087^{1}$.

In addition, we also found that the social guidance provided by the access to peers' progress achieved a higher success rate among all conditions, $F(1,43)=22.246, p<.01, \eta^{2}=.341$. The increase of success rate demonstrates that social guidance indeed helped in guiding the students to the suitable questions. Moreover, students extend their visits on the system and

\footnotetext{
${ }^{1}$ It is an on-going classroom study. The numbers represent the system usage up to date 2011/03/04, which is half way through the semester.
} 
contribute 5.83 sessions on average. The growth of sessions can be attributed to the progress ranking feature, which is intended to create a positively motivating and yet competitive platform. Moreover, in our subjective evaluation [17], most of the students $(84.6 \%)$ appreciated the feature of comparing their progress with others. They liked the interface and found it easy to use (92.3\%). $91.3 \%$ of the students would like to recommend Progressor to their classmates.

\section{CONCLUSION}

In this paper, we presented an improved implementation of the open social student modeling approach based on Parallel IntrospectiveViews interface. This new interface, Progressor, is used to provide access to QuizJET parameterized selfassessment questions in an introductory programming class. The interface allowed the students to visualize not only the student's own model, but also to display parallel views on the models of their peers and the cumulate model of the entire class. Besides, the list of class models are presented and ranked as thumbnails, which allow students to preview their peers' models in an organized manner. Such visualization motivated students to work on the self-assessment questions and extended their visits on the system dramatically.

Moreover, the social features provided by the interface were used for progress comparison, navigation and competition. We observed that the Parallel IntrospectiveViews interface caused the increase of all usage parameters in comparison to a regular portal-based access. However, the Progressor interface allowed the student to achieve the highest success rate in answering the questions among all conditions.

In summary, considering Progressor is still being used in an on-going classroom, which has already produced impressive motivational effects on system usage and social guidance. A deeper analysis on students' learning as well as the privacy management will be followed up. Overall, we believe that the open social student modeling is an interesting and important approach to promote motivation in social learning. We plan to perform more exhaustive evaluation in the future and hope to uncover more motivational effects and learning performance outcome.

\section{ACKNOWLEDGEMENT}

This research was supported in part by the National Science Foundation under Grant No. 0447083.

\section{REFERENCES}

[1] Bull, S. (2004) Supporting learning with open learner models. In: Proceedings of 4th Hellenic Conference on Information and Communication Technologies in Education, Athens, Greece, September 29 - October 3, 2004, pp. 47-61
[2] Mitrovic, A., Martin, B.: Evaluating the Effect of Open Student Models on Self- Assessment. Int. J. of Artificial Intelligence in Education 17(2), 121-144 (2007)

[3] Zapata-Rivera, J.D., Greer, J.E., (2004). Visualizing and inspecting Bayesian belief models. In: International Journal of Artificial Intelligence in Education IJCAI, 14, pp. 1-37. IOS Press.

[4] Vassileva, J. Toward Social Learning Environments. IEEE Transactions on Learning Technologies, 2008, 1(4), p. 199-214.

[5] Dimitrova, V., Self, J., Brna, P . (2001) Applying Interactive Open Learner Models to Learning Technical Terminology. Proceedings of User Modeling'2001, Springer, pp. 148-157.

[6] Chen, Z.H., Chou, C.Y., Deng, Y.C., and Chan, T.W. (2007). Active Open Learner Models as Animal Companions: Motivating Children to Learn through Interaction with My-Pet and Our-Pet, International Journal of Artificial Intelligence in Education, 17(3): 217-226

[7] Bull, S. \& Kay, J. (2007). Student Models that Invite the Learner In: The SMILI Open Learner Modelling Framework, International Journal of Artificial Intelligence in Education 17(2), 89-120.

[8] Vassileva J., Sun L. (2007) Using Community Visualization to Stimulate Participation in Online Communities.e-Service Journal, 6 (1), 3-40.

[9] Bull, S. \& Britland, M. (2007). Group Interaction Prompted by a Simple Assessed Open Learner Model that can be Optionally Released to Peers, in P. Brusilovsky, M. Grigoriadou \& K. Papanikolaou (eds), Proceedings of Workshop on Personalisation in E-Learning Environments at Individual and Group Level, User Modeling 2007.

[10] Mazza, R. \& Dimitrova, V. (2007). CourseVis: a graphical student monitoring tool for supporting instructors in web-based distance courses. International Journal of Human-Computer Studies, 65, 2, 125-139.

[11] Hsiao, I., Sosnovsky,S. and Brusilovsky, P. (2010) Guiding Students to the Right Questions: Adaptive Navigation Support in an E-learning System for Java Programming, Journal of Computer Assisted Learning, Volume 26 Issue 4, Pages 270 - 283.

[12] Bakalov, F., König-Ries, B., Nauerz, A., and Welsch, M. IntrospectiveViews: an Interface for Scrutinizing Semantic User Models. In Proc. of the 18th Intl. Conf on User Modeling, Adaptation and Personalization (2010).

[13] Bakalov, F., König-Ries, B., Nauerz, A., and Welsch, M. Scrutinizing User Interest Models with IntrospectiveViews. In Adjunct Proc. of the 18th Intl. Conf on User Modeling, Adaptation and Personalization (2010).

[14] Bakalov, F., Hsiao, I., Brusilovsky, P., and König-Ries, B. Visualizing Student Models for Social Learning with Parallel IntrospectiveViews. In Proc. of the Workshop on Visual Interfaces to the Social and Semantic $\mathrm{Web}$ at the International Conference on Intelligent User Interfaces (2011).

[15] Vassileva, J.: Open Group Learner Modeling, Interaction Analysis and Social Visualization. In: Dimitrova, V., Tzagarakis, M., Vassileva, J. (eds.) Proceedings of Workshop on Adaptation and Personalisation in Social Systems: Groups, Teams, Communities. Held in conjunction with UM 2007, (2007)

[16] Bandura, A. (1977). Social Learning Theory. New York: General Learning Press.

[17] Hsiao, I., Bakalov, F., Brusilovsky, P., and König-Ries, B. Open Social Student Modeling: Visualizing Student Models with Parallel IntrospectiveViews. In Proc. of the 19th Intl. Conf on User Modeling, Adaptation and Personalization (2011). 